\title{
JAK2V617F variant allele frequency $>50 \%$ identifies patients with polycythemia vera at high risk for venous thrombosis
}

\author{
Paola Guglielmelli (iD ${ }^{1,6}$, Giuseppe G. Loscocco (iD ${ }^{1,6}$, Carmela Mannarelli ${ }^{1}$, Elena Rossi ${ }^{2,3}$, Francesco Mannelli (iD ${ }^{1}$, Francesco Ramundo ${ }^{2}$, \\ Giacomo Coltro ${ }^{1}$, Silvia Betti ${ }^{3}$, Chiara Maccari ${ }^{1}$, Sara Ceglie ${ }^{2}$, Patrizia Chiusolo (D) $^{2,3}$, Chiara Paoli $^{1}$, Tiziano Barbui (iD ${ }^{4}$, Ayalew Tefferi (iD ${ }^{5}$, \\ Valerio De Stefano (iD ${ }^{2,3,6}$ and Alessandro M. Vannucchi (iD ${ }^{1,6}$ 厛
}

(c) The Author(s) 2021

\begin{abstract}
Arterial (AT) and venous (VT) thrombotic events are the most common complications in patients with polycythemia vera (PV) and are the leading causes of morbidity and mortality. In this regard, the impact of JAK2V617F variant allele frequency (VAF) is still debated. The purpose of the current study was to analyze the impact of JAK2V617F VAF in the context of other established risk factors for thrombosis in a total of 8652016 WHO-defined PV patients utilizing two independent cohorts: University of Florence $(n=576)$ as a training cohort and Policlinico Gemelli, Catholic University, Rome $(n=289)$ as a validation cohort. In the training cohort VT free-survival was significantly shorter in the presence of a JAK2V617F VAF $>50 \%$ (HR 4; $p<0.0001$ ), whereas no difference was found for AT (HR 0.9; $p=0.8$ ). Multivariable analysis identified JAK2V617F VAF $>50 \%$ (HR 3.8, $p=0.001)$ and previous VT (HR 2.2; $p=0.04$ ) as independent risk factors for future VT whereas diabetes (HR 2.4; $p=0.02$ ), hyperlipidemia (HR 2.3; $p=0.01)$ and previous AT (HR 2; $p=0.04$ ) were independent risk factors for future AT. Similarly, JAK2V617F VAF $>50 \%(H R 2.4 ; p=0.01)$ and previous VT (HR 2.8; $p=0.005)$ were confirmed as independent predictors of future VT in the validation cohort. Impact of JAK2V617F VAF $>50 \%$ on VT was particularly significant in conventional low-risk patients, both in Florence (HR 10.6, $p=0.005)$ and Rome cohort (HR 4; $p=0.02$ ). In conclusion, we identified JAK2V617F VAF $>50 \%$ as an independent strong predictor of VT, supporting that $A T$ and $V T$ are different entities which might require distinct management.
\end{abstract}

Blood Cancer Journal (2021)11:199; https://doi.org/10.1038/s41408-021-00581-6

\section{INTRODUCTION}

Polycythemia Vera (PV) is a Philadelphia-negative chronic myeloproliferative neoplasm (MPN) along with essential thrombocythemia, overt myelofibrosis (MF) and pre-fibrotic MF. It is mainly characterized by clonal erythrocytosis associated with an increased risk of thrombo-hemorrhagic complications, progression to MF and, to lesser extent, transformation to acute myeloid leukemia (AML). Other disease features which may negatively impact on quality of life include splenomegaly, systemic and microcirculatory symptoms and pruritus. Almost all patients harbor a somatic JAK2 (Janus kinase 2; 9p24) mutation, in more than $95 \%$ of cases located in exon 14 (JAK2V617F), whereas the remaining are heterogeneous insdel changes at exon 12 [1]. Current management of PV relies on a two-tiered model that identifies patients at high-risk (age $\geq 60$ years and/or history of cardiovascular events (CV)) and low-risk (absence of both risk factors) of thrombosis. In addition to low-dose aspirin and phlebotomies to keep hematocrit below $45 \%$, cytoreductive therapy is recommended for high-risk patients [2, 3]. The International Working Group on Myeloproliferative Neoplasms Research and Treatment (IWG-MRT) identified prior arterial events and hypertension as risk factors for arterial thrombosis whereas prior venous events and age $\geq 65$ years were identified as risk factors for venous thrombosis [4], pointing to arterial and venous thrombosis as two biologically different processes with distinct disease risk factors. More recently, other studies focused on additional risk factors for thrombosis in PV including generic CV risk factors and leukocytosis $[5,6]$.

Building upon high-throughput sequencing data from collaborative studies between Mayo Clinic, Rochester, USA and University of Florence, Italy, an integrated genetic and clinical survival risk model for PV (MIPSS-PV) was developed, that included SRSF2 mutations, age $>67$ years, thrombosis history and leukocytosis $(\geq 15 \times 109 / L)$ as independent risk factors for reduced overall survival, whereas no unique mutation profile was associated with an increased risk for thrombosis [7].

In the 16 years since the discovery of JAK2V617F as a driver mutation in MPN, investigators examined the role of JAK2V617F as a risk factor either as a qualitative and quantitative variable for thrombosis. Many studies documented a higher risk for thrombotic events in patients with JAK2V617F mutated ET or MF compared with negative ones; as a consequence, JAK2V617F genotype was incorporated in the International Prognostic Score for Thrombosis in ET $[8,9]$. In PV patients, the JAK2 variant allele frequency (VAF) is

\footnotetext{
${ }^{1}$ Department of Experimental and Clinical Medicine, CRIMM, Center of Research and Innovation of Myeloproliferative Neoplasms, Azienda Ospedaliero-Universitaria Careggi, University of Florence, Florence, Italy. ${ }^{2}$ Section of Hematology, Department of Radiological and Hematological Sciences, Catholic University, Rome, Italy. ${ }^{3}$ Fondazione Policlinico Universitario A. Gemelli IRCCS, Rome, Italy. ${ }^{4}$ FROM Research Foundation, ASST Papa Giovanni XXIII, Bergamo, Italy. ${ }^{5}$ Divisions of Hematology, Mayo Clinic, Rochester, MN, USA. ${ }^{6}$ These authors contributed equally: Paola Guglielmelli, Giuseppe G. Loscocco, Valerio De Stefano, Alessandro M. Vannucchi. ${ }^{\otimes}$ email: a.vannucchi@unifi.it
} 
highly heterogeneous at diagnosis, with median value around $50 \%$, and correlates with some phenotypic traits; in a previous study a VAF $>75 \%$ was associated with higher rate of thrombotic events after diagnosis [10], and a recent study including a large cohort of Chinese patients reported a 4.6 times higher incidence of thrombosis in PV patients with JAK2V617F VAF $\geq 50 \%$ [11]. Moreover, a JAK2V617F VAF $>50 \%$ was also associated with increased fibrotic transformation [12]. At the light of recent observations, it seems that not only circulating blood cells, but also the vessels can participate in thrombosis. Noteworthy, the JAK2V617F mutation has been reported in endothelial cells in some MPN patients, especially those with thrombosis [13, 14], raising the question of their thrombogenicity. Moreover, it is still unclear why splanchnic vein thrombosis (SVT), including hepatic veins, portal veins, splenic veins, or mesenteric veins are a privileged site of thrombosis during MPN, particularly in PV patients, and it raises the hypothesis of a specific physiopathology. SVT are more common in young female patients and in those with a low JAK2V617F allele burden [15-17], whereas in a recent paper a JAK2-mutant allele burden $\geq 50 \%$ and the presence of chromatin/ spliceosome/TP53 mutations identified high-risk SVT-MPN patients with a worse event-free (including both AML and MF progression) and overall survival at 10 years [18].

However, whether the JAK2V617F VAF is prognostically meaningful, particularly concerning thrombosis risk in PV patients, is still debated. The aim of the current study was to evaluate the impact of JAK2 V617F VAF at diagnosis on rate of arterial and venous thrombosis (with the exclusion of SVT from the latter), in the context of other established risk factors, in 2016 WHO-defined PV patients belong to a training cohort collected from Center of Research and Innovation in Myeloproliferative Neoplasms (CRIMM), University of Florence, Italy and a validation cohort from Policlinico Gemelli, Catholic University, Rome, Italy.

\section{MATERIALS AND METHODS \\ Study population}

This retrospective study was approved by the Institutional review board by the Local Ethics Committee at University of Florence and Policlinico Gemelli, Catholic University, Rome. The study was conducted in accordance with the ethical guidelines of the Declaration of Helsinki. Study patients were selected from respective institutional databases provided they were confirmed according to the 2016 WHO criteria for PV diagnosis and written, informed consent was obtained from all living subjects; treatment approaches were in accordance with standard of care at the time of initial diagnosis. Diagnostic criteria for leukemic and myelofibrotic transformation were revised according to the 2016 WHO classification [19] and international working group for MPN research and treatment (IWG-MRT) criteria [20], respectively. JAK2 exon 12 mutated PV patients were excluded, and all patients were annotated for JAK2V617F VAF, determined within 3 years from diagnosis, according to previous published methods [21]. Bone marrow biopsies were internally reviewed to adhere to current criteria and in those without an evaluable bone marrow histology, PV diagnosis relied on the presence of both elevated hemoglobin levels (i.e., $>16.5 / 16.0 \mathrm{~g} / \mathrm{dl}$ in males and females, respectively), presence of JAK2V617F mutation and low serum erythropoietin, according to the latest WHO criteria. Arterial thrombosis included ischemic stroke, transient ischemic attack, retinal artery occlusion, coronary arterial disease, and peripheral arterial disease, whereas venous thrombosis included cerebral venous thrombosis, deep vein thrombosis of the limbs, pulmonary embolism and superficial vein thrombosis. All reported thrombotic events were objectively identified by imaging, serological biomarkers and/or electrocardiogram. Thrombotic events were considered as post diagnosis if occurring at least 4 weeks after PV diagnosis whereas thrombotic events before diagnosis included all events that occurred at any time prior to diagnosis. Microcirculatory symptoms included dizziness, headaches, visual disturbances, erythromelalgia, distal paresthesia and acrocyanosis. Major bleedings were defined based on International Society on Thrombosis and Hemostasis (ISTH) [22] definition as: gastrointestinal, internal organ, intraarticular, cerebrovascular, retroperitoneal bleed or any bleeding requiring medical and/or surgical intervention, hospitalization and/or resulting in death.

\section{Statistical methods}

For the purposes of the current study, only the first arterial and venous event occurring after PV diagnosis was considered. Continuous variables were summarized as median and minimum-maximum ranges. Distribution of continuous variables was compared using nonparametric test (Mann-Whitney), while nominal variables were compared with the Chisquare test. Cox proportional hazard regression model was used for univariate and multivariable analysis for arterial and venous thrombosisfree survival. Survival analysis was considered from the date of diagnosis to date of death or last contact. Thrombosis-free, leukemia-free and myelofibrosis-free survival calculations considered the event as the uncensored variable. The Kaplan-Meier method was used to construct time-to-event curves, which were compared by log-rank test, whereas a receiver operating characteristics curve (ROC) was used to determine the best thresholds of VAF using area under curve (AUC) estimate through Youden index analysis. For all tested hypotheses, two-tailed $p$ values less than 0.05 were considered significant. All the statistical analyses were performed with SPSS software, version 27 (IBM-Corp), JMP Pro 15.1 .0 software from SAS Institute (Cary, NC) and Statistical Package R version 4.1.1.

\section{RESULTS}

\section{Patient characteristics of the training cohort}

Among a total of 576 patients from the University of Florence training cohort (seen 1981-2020), median age at diagnosis was 61.4 years (range, $18-92$ ) and $58.2 \%$ were male; $60.4 \%$ were at high-risk for thrombosis based on current risk stratification. Median JAK2V617F VAF was $41.5 \%$ (range, 0.3-100). Leukocytosis $\left(\geq 11 \times 10^{9} / \mathrm{L}\right)$ was documented in $37.9 \%$ of patients, whereas palpable splenomegaly, microcirculatory symptoms, constitutional symptoms, and pruritus were reported in $35.7 \%(n=194), 31.2 \%$ $(n=170), 12.2 \% \quad(n=69)$ and $39.5 \% \quad(n=223)$ of patients, respectively. The incidence of cardiovascular (CV) risk factors at diagnosis was as follows: hypertension (56\%), diabetes (10.3\%), hyperlipidemia (15.9\%), and active smoking (16\%). With a median survival of 21.7 years, $13 \%$ and $2.1 \%$ of patients experienced myelofibrotic and leukemic transformation during the course of disease, respectively; $16.7 \%$ of patients died. Clinical and laboratory patients' characteristics are detailed in Table 1.

A total of $76(13.2 \%)$ patients had an arterial thrombotic event before or coincident with PV diagnosis, whereas 49 (8.5\%) patients had at least one arterial event during follow-up. As regards venous thrombotic events, $52(9 \%)$ and $39(6.8 \%)$ patients had a venous thrombotic event before/at or after PV diagnosis, respectively. Overall a total of 88 thrombotic events occurred in 78 patients $(13.5 \%), 1.7 \%$ of whom having experienced both an arterial and venous thrombosis. Arterial events occurred before/at PV diagnosis were mainly represented by cerebrovascular and cardiovascular events. Ischemic stroke, transient ischemic attack and acute myocardial infarction occurred in 22 (28.9\%), 17 (22.4\%) and 26 (34.2\%) patients, respectively. Similarly, the most common arterial thrombosis during follow-up were transient ischemic attack $(n=$ $15 ; 30.6 \%)$, followed by acute myocardial infarction ( $n=13 ; 26.5 \%)$ and ischemic stroke $(n=7 ; 18 \%)$. Concerning venous events, deep vein thrombosis, superficial vein thrombosis and pulmonary embolism, represented the most common events both before/at $(53.8 \%, 17.3 \%$ and $15.4 \%$, respectively) and after $(48.7 \%, 20.5 \%$ and $17.9 \%$, respectively) diagnosis. Overall, the incidence rate of thrombosis was $0.83 \%$ /year for VT and $1.04 \%$ /year for AT. In Table 2 the types of arterial and venous thrombosis occurred before/at or after PV diagnosis are detailed.

\section{Risk factors for thrombosis in training cohort}

Considering the patients included in the Florence cohort we found that JAK2V617F VAF as a continuous variable was correlated with the risk of venous thrombosis after diagnosis $(p=0.003 ; \mathrm{HR} 1 ; 95 \%$ $\mathrm{Cl} 1-1.1)$ but not with arterial thrombosis $(p=0.8 ; \mathrm{HR} 1 ; 95 \% \mathrm{Cl}$ $0.9-1)$. A receiver operating characteristic (ROC) curve was used to determine the best cut-off level for JAK2V617F VAF predicting 
Table 1. Laboratory and clinical characteristics of JAK2V617F positive PV patients from training cohort stratified by their variant allele frequency (VAF $>/ \leq 50 \%$ ).

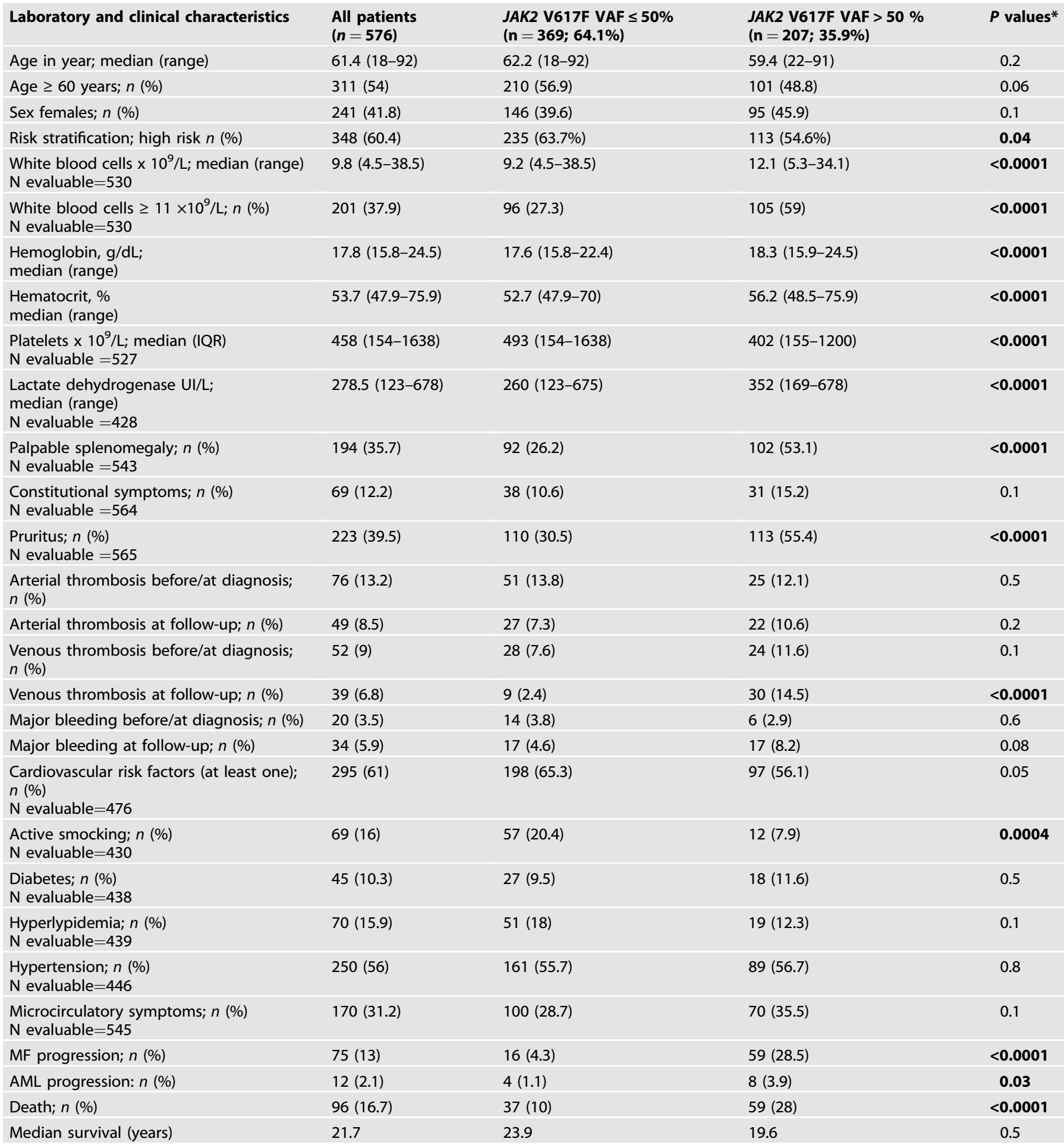

* Significant $p$ values highlighted in bold refer to comparison of VAF $\leq 50 \%$ and $>50 \%$.

venous thrombosis; the curve showed an area under curve (AUC) of 0.72 (Supplementary Fig. 1), and the best cut-off value was $\mathrm{VAF}=50 \%$. Accordingly, we divided PV patients in those with $\mathrm{VAF} \leq 50 \%(369 ; 64.1 \%)$ and $>50 \%(207 ; 35.9 \%)$. Compared to patients with $J A K 2 \mathrm{~V} 617 \mathrm{~F} \mathrm{VAF} \leq 50 \%$, those with $\mathrm{VAF}>50 \%$ displayed higher white blood cells count $(p<0.0001)$, higher hematocrit and hemoglobin levels ( $p<0.0001$ each), lower platelet count $(p<0.0001)$, had more commonly palpable splenomegaly $(p<0.0001)$ and pruritus $(p<0.0001)$. A comparison of laboratory and clinical variables in patients stratified by their VAF ( $>$ vs $\leq 50 \%$ ) is outlined in Table 1 . The rate of venous thrombosis during follow-up was significantly higher in the 
Table 2. Type of thrombotic events occurring before/at diagnosis or during follow-up in training cohort.

\begin{tabular}{|lll|}
\hline University of Florence cohort $(\boldsymbol{n}=\mathbf{5 7 6})$ & \multicolumn{1}{l}{$\begin{array}{l}\text { Before/at } \\
\text { diagnosis }\end{array}$} & $\begin{array}{l}\text { During } \\
\text { follow-up }\end{array}$ \\
\hline Arterial thrombosis $(\boldsymbol{n}, \%)$ & $n=76$ & $n=49$ \\
\hline Acute myocardial infarction & $26(34.2)$ & $13(26.5)$ \\
\hline Unstable angina & $4(5.3)$ & $5(10.2)$ \\
\hline Stroke & $22(28.9)$ & $12(24.5)$ \\
\hline Transient ischemic attack & $17(22.4)$ & $15(30.6)$ \\
\hline Peripheral arterial thrombosis & $5(6.6)$ & $4(8.2)$ \\
\hline Abdominal thrombosis & $1(1.3)$ & - \\
\hline Retinal thrombosis & $1(1.3)$ & - \\
\hline Venous thrombosis $(\boldsymbol{n}, \%)$ & $n=52$ & $n=39$ \\
\hline Deep vein thrombosis & $28(53.8)$ & $19(48.7)$ \\
\hline Pulmonary embolism & $8(15.4)$ & $7(17.9)$ \\
\hline $\begin{array}{l}\text { Pulmonary embolism }+ \text { deep } \\
\text { vein thrombosis }\end{array}$ & $1(1.9)$ & $2(5.1)$ \\
\hline Cerebral vein thrombosis & $3(5.8)$ & $1(2.6)$ \\
\hline Superficial vein thrombosis & $9(17.3)$ & $8(20.5)$ \\
\hline Retinal thrombosis & $3(5.8)$ & $2(5.1)$ \\
\hline
\end{tabular}

presence of a JAK2V617F VAF $>50 \%(2.4 \%$ vs $14.5 \% ; p<0.0001)$, that was associated with a significantly worse thrombosis free survival HR 4, 95\% Cl 1.9-8.6, $p<0.0001$; (Fig. 1A). Conversely, no difference was found for arterial thrombosis (HR 0.9, 95\% Cl 0.5$1.6, p=0.7$; Fig. 1B). In addition to $\mathrm{VAF}>50 \%$, univariate analysis for venous thrombosis-free survival (V-TFS) identified a previous venous thrombosis (HR 2.9,95\% Cl 1.4-6.1; $p=0.006$ ), leukocytosis $\geq 11 \times 109 / \mathrm{L}$ (HR 1.9; $95 \% \mathrm{Cl} 1.1-3.4 ; p=0.02)$ and palpable splenomegaly (HR 1.9,95\% Cl 1-3.6; $p=0.04$ ) as risk factors for venous thrombosis. Age $\geq 60$ years was not significant (HR 1,95\% $\mathrm{Cl} 0.9-1 ; p=0.9)$. Multivariable analysis confirmed $\mathrm{VAF}>50 \%$ (HR $3.8,1.7-8.6 ; p=0.001$ ) and previous venous thrombosis (HR 2.2, $95 \% \mathrm{Cl} 1.1-5 ; p=0.04)$ as independent risk factors for future venous thrombosis. In contrast, univariate analysis for arterial thrombosis-free survival (A-TFS) identified history of arterial thrombosis (HR 2.5, 95\% Cl 1.3-4.9; $p=0.007$ ), diabetes (HR 3.3; 95\% Cl 1.6-6.5; $p=0.0007$ ), hyperlipidemia (HR 3.1, 95\% Cl 1.7-5.6; $p=0.0003$ ) and hypertension (HR 2, 95\% Cl 1-3.9; $p=0.04$ ) as predictors of a subsequent arterial event; age $\geq 60$ years showed only a trend for significance (HR 1.7; $95 \% \mathrm{Cl} 0.9-3.1, p=0.08)$. Multivariable analysis for A-TFS identified diabetes (HR $2.4 ; 95 \% \mathrm{Cl}$ $1.2-5, p=0.02$ ), hyperlipidemia (HR 2.3; $9 \% \mathrm{Cl} 1.2-4.3, p=0.01$ ) and previous arterial event (HR 2.1; $95 \% \mathrm{Cl} 1-4.2, p=0.04)$ as independent predictors of a subsequent arterial event. Univariate and multivariable analysis, considering clinical and laboratory variables, for both venous and arterial thrombosis-free survival among patients from the Florence cohort are reported in Table 3.

Finally, in order to mitigate the confounding effect of different therapeutic approach concerning the impact of VAF $>50 \%$ on VTFS, patients were stratified according to the conventional risk category attributed at diagnosis, which correlated with cytoreductive therapy (high risk, $>90 \%$ cases received hydroxyurea) or phlebotomy only (in the majority of low risk patients) along with low-dose acetylsalicylic acid (ASA); the Kaplan-Meier curves presented in Fig. $2 \mathrm{~A}$ and $\mathrm{B}$ show that a JAK2V617F VAF $>50 \%$ remains significant for the risk of future venous events both in low-risk (HR 10.6; 95\% Cl 1.4-81.5, $p=0.005$ ) and high-risk patients (HR 3.5; 95\% Cl 1.5-8.3, $p=0.002$ ), respectively. Moreover, patients with $\mathrm{VAF}>50 \%$ showed a higher risk for MF progression (HR 3.6; $95 \% \mathrm{Cl} 2-6.3 ; p<0.0001)$ without any differences in leukemic
A
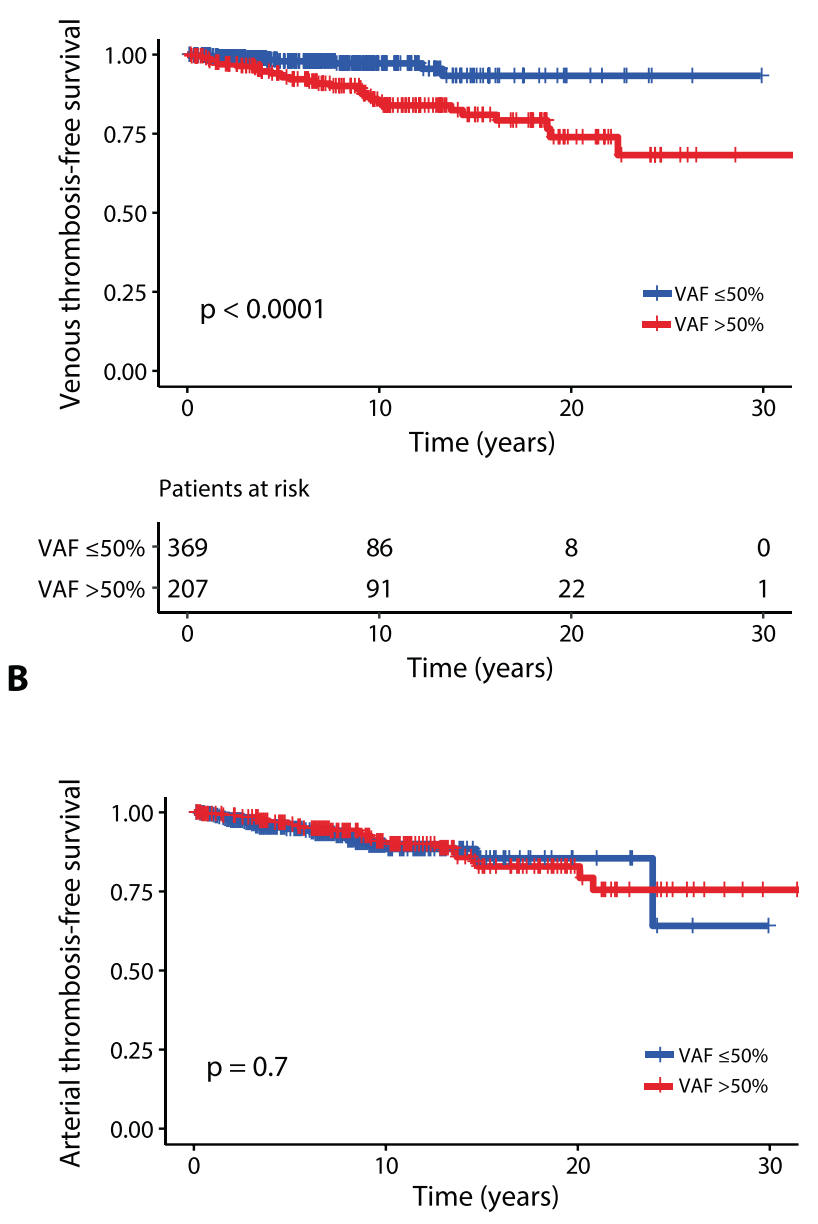

$\begin{gathered}\text { Patients at risk } \\
\mathrm{VAF} \leq 50 \% \\
\mathrm{VAF}>50 \%\end{gathered}-$\begin{tabular}{cccc}
369 & 82 & 7 & 0 \\
207 & 97 & 23 & 2 \\
\hline 0 & 10 & 20 & 30
\end{tabular}

Fig. 1 Venous and arterial thrombosis-free survival for JAK2V617F positive PV patients from training cohort stratified by their JAK2V617F VAF ( $>\mathbf{5 0} \%$ vs $\leq \mathbf{5 0} \%$ ). Kaplan-Meier curves representing venous thrombosis-free survival (A) and arterial thrombosis-free survival (B) including a total of 576 PV patients. The number of patients at risk for each time point is shown below the graph. Tick marks indicate censored data.

progression (HR 1.1; 95\% Cl 0.3-3.7; $p=0.9$ ) and overall survival (HR $1.1 ; 95 \% \mathrm{Cl} 0.7-1.8 ; p=0.5$ ), Supplementary Fig. 2A-C.

Patient characteristics of the validation cohort

In the Policlinico Gemelli, Catholic University database a totally of 289 PV patients, with confirmed 2016 WHO criteria, who were fully annotated for JAK2V617F VAF were included in the current analysis.

Median age at diagnosis was 61 years (range, 19-89) and $49.5 \%$ were male; $57.8 \%$ were at high-risk for thrombosis based on current risk stratification. Median JAK2 VAF was $51 \%$ (range, $1-100)$. Leukocytosis $\left(\geq 11 \times 10^{9} / \mathrm{L}\right)$ was documented in $35.1 \%$ of patients, whereas palpable splenomegaly, microcirculatory symptoms, constitutional symptoms, and pruritus were reported in $35.1 \%(n=97), 38.4 \%(n=111), 11.7 \%(n=33)$ and $38.9 \%(n=$ $110)$ of patients, respectively. 
Table 3. Univariate and multivariable analysis for venous and arterial thrombosis-free survival among PV patients from training cohort.

\begin{tabular}{|c|c|c|c|c|}
\hline \multirow{2}{*}{$\begin{array}{l}\text { Clinical and laboratory } \\
\text { variables }\end{array}$} & \multicolumn{2}{|c|}{ Venous thrombosis-free survival } & \multicolumn{2}{|c|}{ Arterial thrombosis-free survival } \\
\hline & $\begin{array}{l}\text { Univariate analysis HR } \\
(95 \% \mathrm{CI}) P^{*}\end{array}$ & $\begin{array}{l}\text { Multivariable analysis HR } \\
(95 \% \mathrm{Cl}) P^{*}\end{array}$ & $\begin{array}{l}\text { Univariate analysis HR } \\
(95 \% \mathrm{CI}) P^{*}\end{array}$ & $\begin{array}{l}\text { Multivariable analysis HR } \\
(95 \% \mathrm{CI}) P^{*}\end{array}$ \\
\hline Age in years & $1(0.9-1) ; 0.6$ & & $1(1-1.1) ; 0.04$ & $1(1-1.1) ; 0.3$ \\
\hline Age $\geq 60$ years & $1(0.5-1.9) ; 0.9$ & & $1.7(0.9-3.1) ; 0.08$ & \\
\hline Male sex & $0.7(0.4-1.4) ; 0.3$ & & $1.4(0.8-2.5) ; 0.2$ & \\
\hline $\begin{array}{l}\text { High-risk } \\
\text { (conventional risk } \\
\text { stratification) }\end{array}$ & $1.6(0.8-3.2) ; 0.1$ & & $1.6(0.9-2.9) ; 0.1$ & \\
\hline $\begin{array}{l}\text { White blood cells } \\
\left(\times 10^{9} / \mathrm{L}\right)\end{array}$ & $1(1-1.1) ; 0.1$ & & $1(0.9-1) ; p=0.5$ & \\
\hline $\begin{array}{l}\text { White blood cells } \\
(\geq 11 \times 109)\end{array}$ & $1.9(1.1-3.4) ; 0.02$ & $0.9(0.5-1.9) ; 0.9$ & $0.8(0.5-1.5) ; 0.5$ & \\
\hline Hemoglobin (g/dL) & $1(0.8-1.3) ; 0.7$ & & $0.9(0.7-1.1) ; 0.3$ & \\
\hline $\begin{array}{l}\text { JAK2 V617F } \\
\text { VAF (\%) (continuous variable) }\end{array}$ & $1(1-1.1) ; 0.003$ & & $1(0.9-1) ; 0.9$ & \\
\hline $\begin{array}{l}J A K 2 \text { V617F } \\
V A F>50 \%\end{array}$ & $4(1.9-8.6) ; 0.0003$ & $3.8(1.7-8.6) ; 0.001$ & $0.9(0.5-1.6) ; 0.7$ & \\
\hline Palpable splenomegaly & $1.9(1-3.6) ; 0.04$ & $1.3(0.7-2.7) ; 0.3$ & $1(0.6-1.9) ; 0.8$ & \\
\hline $\begin{array}{l}\text { Arterial thrombosis before/at } \\
\text { diagnosis }\end{array}$ & $1.7(0.8-1.4) ; 0.2$ & & $2.5(1.3-4.9) ; 0.007$ & $2.1(1-4.1) ; 0.04$ \\
\hline $\begin{array}{l}\text { Venous thrombosis before/at } \\
\text { diagnosis }\end{array}$ & $2.9(1.4-6.1) ; 0.006$ & $2.2(1.1-5) ; 0.04$ & $0.5(0.2-1.7) ; 0.3$ & \\
\hline $\begin{array}{l}\text { Major bleeding before/at } \\
\text { diagnosis }\end{array}$ & $0.05(1-460) ; 0.5$ & & $0.05(0.1-120) ; 0.5$ & \\
\hline
\end{tabular}

*Significant $p$ values are highlighted in bold.

$P V$ polycythemia vera, HR hazard ratio, VAF variant allele frequency, $C l$ confidence interval.

Among CV risk factors, hypertension, diabetes, hyperlipidemia, and active smoking were reported in $47.4 \%, 5.9 \%, 14.9 \%$ and $17.6 \%$ of patients, respectively. Median survival of the whole patients' cohort was not reached and during the course of disease, $7.9 \%$ and $1.7 \%$ of the patients experienced myelofibrotic and leukemic progression, respectively; $6.6 \%$ of patients died. Clinical and laboratory patients' characteristics of the Rome cohort are detailed in Table 4.

In the validation cohort, the incidence of arterial thrombosis was $17.3 \%(n=50)$ before/at diagnosis and $15.6 \%(n=45)$ during follow-up. The most common arterial events were represented by cerebrovascular thrombosis $(57.9 \%$ and $58.8 \%$ at diagnosis and during follow-up, respectively) followed by cardiovascular events (39.5\% and $26.8 \%$ at diagnosis and during follow-up, respectively). Regarding venous thrombosis, 36 events (incidence of 12.5\%) were reported before/at diagnosis and 43 events (incidence of $14.9 \%)$ during follow-up. Deep vein thrombosis and pulmonary embolisms were more common at diagnosis (44.5\% and $22.2 \%$ ), whereas superficial vein thrombosis were more common during follow-up (46.5\%). Overall, the incidence rate of thrombosis was $1.92 \% /$ year for VT and $2.08 \%$ /year for AT.

The types of arterial and venous events occurred both at diagnosis (data on type of events occurred before diagnosis were not available) or during follow-up in validation cohort are outlined in Supplementary Table 1.

\section{Validation of risk factors for thrombosis}

Confirming findings in the training cohort, the rate of venous thrombosis during follow-up was significantly higher in the presence of a JAK2V617F VAF $>50 \%(\mathrm{HR} 2.9,95 \% \mathrm{Cl} 1.2-4.2 ; p=$ 0.007); (Fig. 3A), whereas no difference was found for arterial thrombosis (HR 1, 95\% Cl 0.6-1.9, $p=0.9$; Fig. 3B). A comparison of laboratory and clinical variables stratifying patients by their VAF ( $>50 \%$ vs $\leq 50 \%$ ) is detailed in Table 4 . In addition to VAF $>50 \%$, univariate analysis for V-TFS identified previous venous thrombosis (HR 5.1, 95\% Cl 1.8-14.2; $p=0.002$ ) as risk factors for future venous thrombosis. Multivariable analysis confirmed JAK2V617F $\mathrm{VAF}>50 \%$ (HR 2.4, 95\% Cl 1.2-4.8; $p=0.01)$ and previous venous thrombosis (HR 2.8,95\% Cl 1.4-5.7; $p=0.005$ ) as independent risk factors for future venous events. Regarding A-TFS, univariate analysis identified history of arterial thrombosis as a quite significant predictor of future arterial event $(\mathrm{HR} 2.4 ; 95 \% \mathrm{Cl}$ $0.9-6 ; p=0.07$ ). Conversely, age $\geq 60$ years (HR $1.1 ; 95 \% \mathrm{Cl} 0.6-2$; $p=0.7$ ) and presence of CV risk factors at diagnosis (HR 0.8; $95 \%$ $\mathrm{Cl} 0.5-1.5 ; p=0.6$ ) were not significant. Finally, JAK2V617F VAF > $50 \%$ significantly affected V-TFS in low-risk patients (HR $4 ; 95 \% \mathrm{Cl}$ 
A
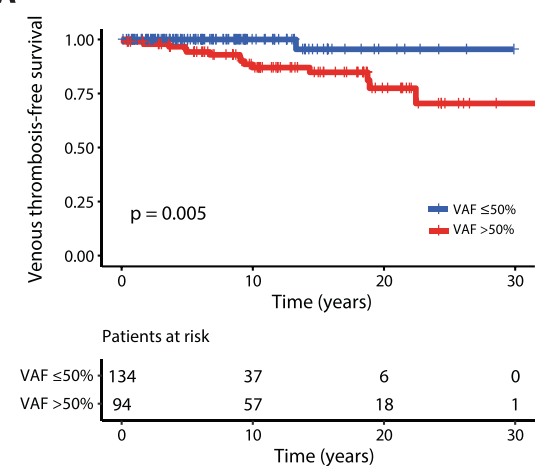

B

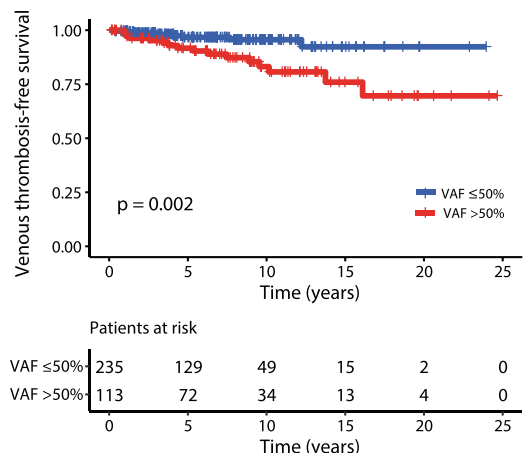

C
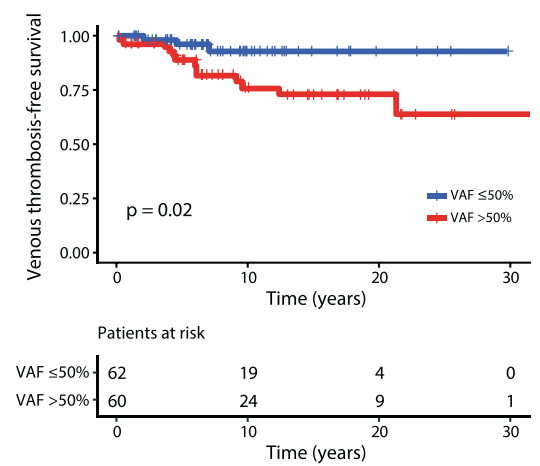

D

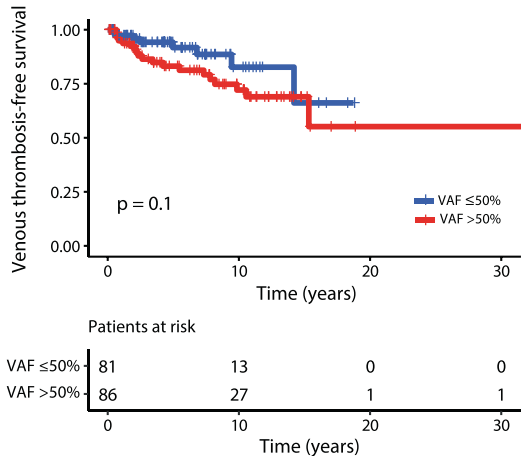

Fig. 2 Venous thrombosis-free survival for low and high-risk JAK2V617F positive PV patients from training and validation cohorts stratified by their JAK2V167F VAF ( $50 \%$ vs $\leq 50 \%)$. Kaplan-Meier curves representing venous thrombosis-free survival for training cohort considering those at low risk (A) and high risk (B) at diagnosis. The same analysis on validation cohort patients at low-risk (C) and high-risk (D). The number of patients at risk for each time point is shown below the graph. Tick marks indicate censored data.

$1.1-13.9, p=0.02$, Fig. 2 C) whereas the impact of VAF $>50 \%$ was close to significance considering high-risk patients' cohort (HR 1.9; $95 \% \mathrm{Cl} 0.8-4.4, p=0.1$, Fig. 2D).

\section{DISCUSSION}

Cardiovascular and thromboembolic events are the most relevant complications in patients with PV and are the major cause of morbidity and mortality. Nowadays, current treatment strategies in PV are directed at preventing thrombotic complications. In this regard, two risk categories are conventionally considered: high (age $\geq 60$ years or thrombosis history) and low (absence of both risk factors). All patients require phlebotomy to keep hematocrit below $45 \%$ and once-daily low-dose ASA, whereas cytoreductive therapy is recommended for high-risk patients. However, despite adherence to treatments, thrombotic risk remains significant at varying degrees during follow-up [23]. A retrospective analysis of patients with MPNs from the Swedish Cancer Registry $(n=9429$; PV, $n=3001$ ), including patients followed from 1987 to 2009, reported that at 3 months after diagnosis, patients with PV had an approximately 3- and 9.7-fold higher risk of arterial thrombosis and venous thrombosis, respectively, compared to controls matched for age and sex [24]. Epidemiological data concerning incidence of arterial and venous thrombosis before/at diagnosis in PV patients derive from two seminal studies. In the European Collaboration on Low-Dose Aspirin in Polycythemia Vera (ECLAP) study arterial and venous thrombosis history before/at diagnosis was documented in $27 \%$ and $11 \%$ of patients, respectively [2]. A significant lower incidence for arterial thrombosis was documented in CYTO-PV trial (arterial 17\%/venous 12\%) that was conducted several years later [25]. Similar results (arterial 16\%/ venous $7.4 \%$ ) were reported in a study including 1545 contemporary PV patients followed in 7 centers in Italy, Austria, and the United States referring to the International Working Group for Myeloproliferative Neoplasms [4]. Prospective and retrospective studies investigated the prognostic role of risk factors for thrombosis in large cohorts of PV patients. However, few data focused on including the prognostic contribution of JAK2V617F VAF on thrombosis in PV patients are available and the role of VAF is still uncertain.

The aim of the current study was to specifically evaluate in 2016 WHO-defined PV patients the impact of JAK2V617F VAF on rate of arterial and venous thrombosis, excluding SVT, in the context of other established risk factors. First, a training cohort from University of Florence was examined. JAK2V617F VAF as a continue variable was correlated with the risk of venous thrombosis after diagnosis $(p=0.003)$ but not with arterial thrombosis $(p=0.8)$. Multivariable analysis identified JAK2V617F VAF $>50 \%$ (HR 3.8, $p=0.001$ ) and previous VT (HR 2.2; $p=0.04$ ) as independent risk factors for future VT whereas diabetes (HR 2.4; $p=0.02$ ), hyperlipidemia (HR 2.3; $p=0.01$ ) and previous AT (HR 2; $p=0.04$ ) were independent risk factors for future AT. JAK2V617F VAF $>50 \%(H R 2.4 ; p=0.01)$ and previous VT (HR 2.8; $p=0.005)$ were confirmed as independent predictors of future VT also in the validation cohort including 289 PV patients from Policlinico Gemelli, Catholic University, Rome. In both cohorts, we confirmed the reported impact of previous venous thrombosis for subsequent venous events and previous arterial thrombosis for arterial events [4, 6]; CV risk factors were significant risk factors for AT in training cohort but not confirmed in validation cohort. Conversely, age was not significant in either cohorts, possibly reflecting changing epidemiology of the disease as well as improvement of general health status and longer life expectation, compared to older studies. From an epidemiological point of view, in the current study including a total of 865 patients, the cumulative incidences of arterial and venous thrombosis before/at diagnosis 
Table 4. Laboratory and clinical characteristics of JAK2V617F positive PV patients from validation cohort stratified by their variant allele frequency (VAF $>/ \leq 50 \%$ ).

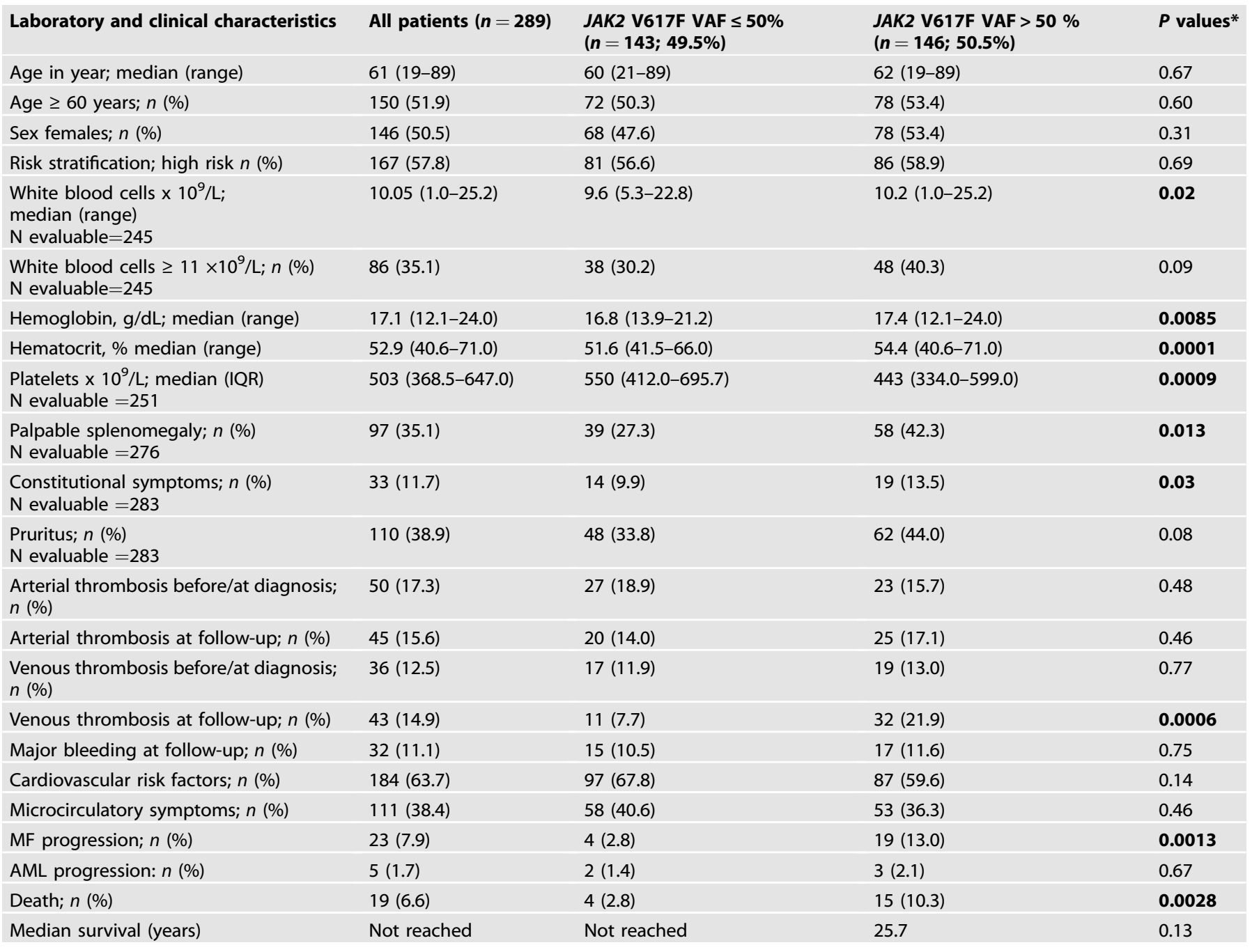

*significant $p$ values highlighted in bold refer to comparison of $\leq 50 \%$ and $>50 \%$.

was $14.6 \%$ and $10.2 \%$ respectively, similarly to the most recent trials reported above $[4,25]$. Considering thrombotic events after diagnosis, the incidence rate was $0.83 \%$ /year for VT and $1.04 \% /$ year for AT in Florence cohort whereas rates were higher in Rome cohort: 1.92\%/year for VT and 2,08\%/year for AT. Pooled incidence rates were $1.36 \% /$ year for AT e $1.18 \% /$ year for VT. Altogether, the frequency of global thrombotic events in our study $(2.4 \% /$ years $)$ was slightly lower than those reported in the Cyto-PV trial $(2.7 \% /$ years) [26]. Earlier diagnosis and treatment, including PV patients fully diagnosed according to the latest 2016 WHO classification, improved management of CV factors and a more appropriate use of phlebotomy, antiplatelet and cytoreductive drugs, might reflect a change of clinical epidemiology of thrombotic events occurring after diagnosis. This notwithstanding, thrombotic risk remains substantial, particularly in those defined at low risk. At this regard, the LOW-PV trial (NCT03003325) is ongoing and evaluating whether the addition of ropeginterferon alfa- $2 b$ to phlebotomy and antiplatelet therapy could improve the outcomes. Interim analysis results documented that ropeginterferon alfa- $2 \mathrm{~b}$ was superior in maintaining the recommended hematocrit value of less than $45 \%$ with a deepest mean change of JAK2V617F VAF at 12 -months follow-up $(-10.43 \%$ vs $-1.03 \% ; p=0.006)$ [27]. More complete and mature data will help to understand how the impact of the reduction of JAK2V617F, if confirmed, may have prognostic implications, in particular on thrombotic risk, in these low-risk patients $[28,29]$.

Recent advances in our understanding of the roles of clonal hematopoiesis, JAK2V617F mutation, endothelial cells and inflammation in thrombosis risk are providing new insights into pathophysiology of PV [30, 31]. In a recent study, RNA sequencing in granulocytes of PV patients documented higher expression levels of $F 3, S E L P, V E G F A$, and $S L C 2 A 1$, that were directly correlated with $J A K 2$ expression and JAK2V617F allele burden in patients with a history of thrombosis [32].

The main potential limitation of our study concerns its retrospective design; moreover, we did not collect data on the use of anticoagulants, dose of antiplatelet therapy, differences in the use of cytoreductive drugs, statins and hypertension treatments along with concomitant thrombophilic disorders, which could confound associations with thrombosis. The intrinsic potential drawbacks of the retrospective design could explain the differences in the incidence rate of thrombosis between the training and validation cohort. However, the study has also strengths regarding the analysis of two large and independent cohorts from Centers with experience in MPNs, the well-defined patient population including a total of 865 PV patients diagnosed 


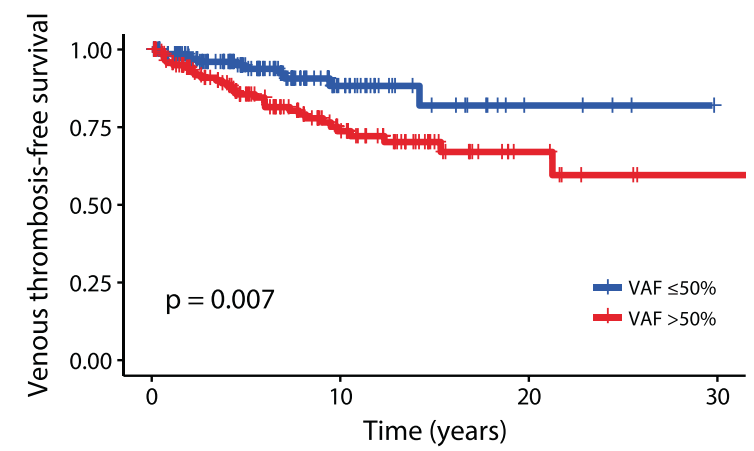

new global thrombotic risk model for PV patients since JAK2V617F VAF correlated with VT but not with AT. However, these findings suggest the classical thrombotic risk model based on age and previous thrombosis may not be enough informative to tailor adequate treatment, especially in the setting of VT prevention. Therefore, in parallel to conventional risk stratification, JAK2V617F VAF might receive consideration for an individualized risk assessment. Whether this should result in modification of the current treatment approach is beyond the scope of this work and would require prospective evaluation.

\section{REFERENCES}

1. Loscocco GG, Guglielmelli $P$, Vannucchi AM. Impact of mutational profile on the management of myeloproliferative neoplasms: a short review of the emerging data. Onco Targets Ther. 2020;13:12367-82.

2. Marchioli R, Finazzi G, Landolfi R, Kutti J, Gisslinger H, Patrono C, et al. Vascular and neoplastic risk in a large cohort of patients with polycythemia vera. J Clin Oncol. 2005;23:2224-32.

3. Barbui T, Tefferi A, Vannucchi AM, Passamonti F, Silver RT, Hoffman R, et al. Philadelphia chromosome-negative classical myeloproliferative neoplasms: revised management recommendations from European LeukemiaNet. Leukemia. 2018;32:1057-69.

B

VAF $\leq 50 \%-$\begin{tabular}{cccc}
143 & 32 & 4 & 0 \\
146 & 51 & 10 & 2 \\
\hline 0 & 10 Time (years) & 20 & 30
\end{tabular}

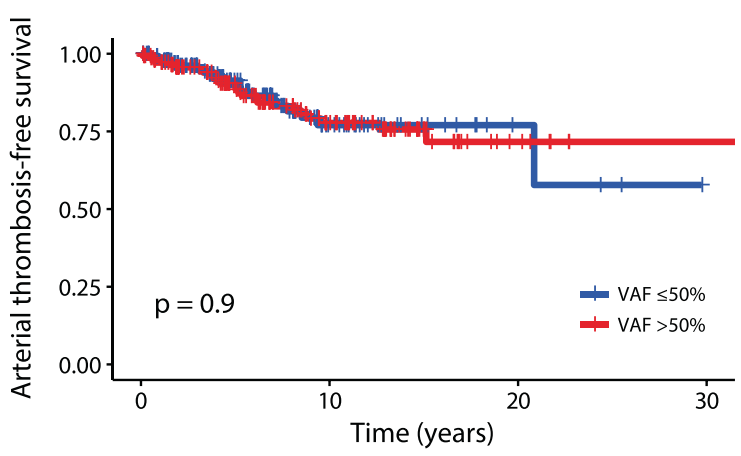

4. Barbui T, Carobbio A, Rumi E, Finazzi G, Gisslinger $H$, Rodeghiero $F$ et al. In contemporary patients with polycythemia vera, rates of thrombosis and risk factors delineate a new clinical epidemiology. Blood. 2014; 124. https://doi.org/ 10.1182/blood-2014-07-591610.

5. Tefferi A, Barbui T. Polycythemia vera and essential thrombocythemia: 2021 update on diagnosis, risk-stratification and management. Am J Hematol. 2020;95:1599-613.

6. Cerquozzi S, Barraco D, Lasho T, Finke C, Hanson CA, Ketterling RP, et al. Risk factors for arterial versus venous thrombosis in polycythemia vera: a single center experience in 587 patients. Blood Cancer J. 2017;7:662.

7. Tefferi A, Guglielmelli P, Lasho TL, Coltro G, Finke CM, Loscocco GG, et al. Mutation-enhanced international prognostic systems for essential thrombocythaemia and polycythaemia vera. Br J Haematol. 2020;189:291-302.

8. Barbui T, Finazzi G, Carobbio A, Thiele J, Passamonti F, Rumi E, et al. Development and validation of an international prognostic score of thrombosis in world health organization-essential thrombocythemia (IPSET-thrombosis). Blood. 2012;120:5128-33. quiz 5252

9. Barbui T, Vannucchi AM, Buxhofer-Ausch V, De Stefano V, Betti S, Rambaldi A, et al. Practice-relevant revision of IPSET-thrombosis based on 1019 patients with WHO-defined essential thrombocythemia. Blood Cancer J. 2015;5:e369.

10. Vannucchi AM, Antonioli E, Guglielmelli P, Longo G, Pancrazzi A, Ponziani V, et al. Prospective identification of high-risk polycythemia vera patients based on JAK2 (V617F) allele burden. Leukemia. 2007;21:1952-9.

11. Zhang Y, Zhou Y, Wang Y, Teng G, Li D, Wang Y, et al. Thrombosis among 1537 patients with JAK2V617F-mutated myeloproliferative neoplasms: risk factors and development of a predictive model. Cancer Med. 2020;9:2096-105.

Fig. 3 Venous and arterial thrombosis-free survival for JAK2V617F positive PV patients from validation cohort stratified by their JAK2V617F VAF ( $>\mathbf{5 0} \%$ vS $\leq \mathbf{5 0 \%}$ ). Kaplan-Meier curves representing venous thrombosis-free survival (A) and arterial thrombosis-free survival (B) including a total of 289 PV patients. The number of patients at risk for each time point is shown below the graph. Tick marks indicate censored data.

according to the latest 2016 WHO classification criteria and managed according to the best-practice principles, the evaluation of patients at diagnosis, and the long follow-up period. Furthermore, due to the low-rate of events and the long followup required, it is unlikely that prospective studies with this primary endpoint might be conducted. In summary, the present study indicates that PV patients with a JAK2V617F VAF > 50\% suffer from increased rate of venous events over time, particularly in conventionally defined low-risk patients. On the other hand, JAK2V617F VAF $>50 \%$ has no impact on arterial thrombosis. Moreover, it confirms the importance of a history of thrombosis in predicting future thrombotic events and supports that arterial and venous events are distinct entities with specific risk factors, including JAK2V617F VAF, that require careful recognition and management. In conclusion, this study does not aim to develop a

12. Passamonti F, Rumi E, Pietra D, Elena C, Boveri E, Arcaini L, et al. A prospective study of 338 patients with polycythemia vera: the impact of JAK2 (V617F) allele burden and leukocytosis on fibrotic or leukemic disease transformation and vascular complications. Leukemia. 2010;24:1574-9.

13. Sozer S, Fiel Ml, Schiano T, Xu M, Mascarenhas J, Hoffman R The presence of JAK2V617F mutation in the liver endothelial cells of patients with Budd-Chiari syndrome. Blood. 2009; 113. https://doi.org/10.1182/blood-2008-11-191544.

14. Rosti V, Villani L, Riboni R, Poletto V, Bonetti E, Tozzi L et al. Spleen endothelial cells from patients with myelofibrosis harbor the JAK2V617F mutation. Blood. 2013; 121. https://doi.org/10.1182/blood-2012-01-404889.

15. Smalberg JH, Arends LR, Valla DC, Kiladjian J-J, Janssen HLA, Leebeek FWG Myeloproliferative neoplasms in Budd-Chiari syndrome and portal vein thrombosis: a meta-analysis. Blood. 2012; 120. https://doi.org/10.1182/blood-2011-09376517.

16. How J, Trinkaus KM, Oh ST Distinct clinical, laboratory and molecular features of myeloproliferative neoplasm patients with splanchnic vein thrombosis. $\mathrm{Br} \mathrm{J}$ Haematol. 2018; 183. https://doi.org/10.1111/bjh.14958.

17. Sant'Antonio E, Guglielmelli $P$, Pieri L, Primignani $M$, Randi $M L$, Santarossa $C$ et al. Splanchnic vein thromboses associated with myeloproliferative neoplasms: An international, retrospective study on 518 cases. Am J Hematol. 2020; 95. https:// doi.org/10.1002/ajh.25677.

18. Debureaux P-E, Cassinat B, Soret-Dulphy J, Mora B, Verger E, Maslah N, et al. Molecular profiling and risk classification of patients with myeloproliferative neoplasms and splanchnic vein thromboses. Blood Adv. 2020;4:3708-15. 
19. Arber DA, Orazi A, Hasserjian R, Thiele J, Borowitz MJ, Le Beau MM, et al. The 2016 revision to the World Health Organization classification of myeloid neoplasms and acute leukemia. Blood. 2016;127:2391-405.

20. Barosi G, Mesa RA, Thiele J, Cervantes F, Campbell PJ, Verstovsek S, et al. Proposed criteria for the diagnosis of post-polycythemia vera and post-essential thrombocythemia myelofibrosis: a consensus statement from the international working group for myelofibrosis research and treatment. Leukemia. 2008;22:437-438.

21. Pacilli A, Rotunno G, Mannarelli C, Fanelli T, Pancrazzi A, Contini E, et al. Mutation landscape in patients with myelofibrosis receiving ruxolitinib or hydroxyurea. Blood Cancer J. 2018;8:122.

22. Schulman S, Kearon C. Definition of major bleeding in clinical investigations of antihemostatic medicinal products in non-surgical patients. J Thromb Haemost. 2005;3:692-694.

23. Tefferi A, Vannucchi AM, Barbui T Polycythemia vera: historical oversights, diagnostic details, and therapeutic views. Leukemia. 2021. https://doi.org/ 10.1038/s41375-021-01401-3.

24. Hultcrantz M, Björkholm M, Dickman PW, Landgren O, Derolf ÅR, Kristinsson SY, et al. Risk for arterial and venous thrombosis in patients with myeloproliferative neoplasms: a population-based cohort study. Ann Intern Med. 2018;168:317-25.

25. Marchioli R, Finazzi G, Specchia G, Cacciola R, Cavazzina R, Cilloni D, et al. Cardiovascular events and intensity of treatment in polycythemia vera. $N$ Engl J Med. 2013:368:22-33.

26. Vannucchi AM, Antonioli E, Guglielmelli P, Rambaldi A, Barosi G, Marchioli R, et al Clinical profile of homozygous JAK2 $617 \mathrm{~V}>\mathrm{F}$ mutation in patients with polycythemia vera or essential thrombocythemia. Blood. 2007;110:840-846.

27. Barbui T, Vannucchi AM, De Stefano V, Masciulli A, Carobbio A, Ferrari A et al. Ropeginterferon alfa- $2 b$ versus phlebotomy in low-risk patients with polycythaemia vera (Low-PV study): a multicentre, randomised phase 2 trial. Lancet Haematol. 2021; 8. https://doi.org/10.1016/S2352-3026(20)30373-2.

28. Kiladjian JJ, Barbui T. From leeches to interferon: should cytoreduction be prescribed for all patients with polycythemia vera? Leukemia 2020;34:2837-2839.

29. Heibl S. Is it time for interferon in low-risk patients with polycythaemia vera? Lancet Haematol. 2021;8:e162-e163.

30. Guy A, Poisson J, James C. Pathogenesis of cardiovascular events in BCR-ABL1negative myeloproliferative neoplasms. Leukemia. 2021;35:935-55.

31. Moliterno AR, Ginzburg YZ, Hoffman R. Clinical insights into the origins of thrombosis in myeloproliferative neoplasms. Blood. 2021;137:1145-53.

32. Gangaraju R, Song J, Kim SJ, Tashi T, Reeves BN, Sundar KM, et al. Thrombotic, inflammatory, and HIF-regulated genes and thrombosis risk in polycythemia vera and essential thrombocythemia. Blood Adv. 2020;4:1115-30.

\section{ACKNOWLEDGEMENTS}

This work has received financial support from Associazione Italiana per la Ricerca sul Cancro (AIRC) $5 \times 1000$ call "Metastatic disease: the key unmet need in oncology" to MYNERVA (MYeloid NEoplasms Research Venture AIRC), project \#21267; Bando Ricerca Finalizzata Ministero della Salute RF-2016-02362930.

\section{AUTHOR CONTRIBUTIONS}

PG, GGL, AT, VDS, and AMV designed research, analyzed and interpreted data. PG, $\mathrm{CM}, \mathrm{ER}, \mathrm{FM}, \mathrm{FR}, \mathrm{GC}, \mathrm{SB}, \mathrm{ChM}, \mathrm{SC}, \mathrm{PC}, \mathrm{CP}, \mathrm{TB}$ contributed analytical tools and data. PG, GGL, VDS, FR performed statistical analysis; PG, GGL, AMV wrote the manuscript. All authors participated in collecting, analyzing, interpreting the data, checked and approved the final version of the manuscript.

\section{COMPETING INTERESTS}

PG received personal fees for advisory board and/or lectures from Novartis. VDS received personal fees for advisory board and/or lectures from AbbVie, AOP Orphan Pharmaceutical, and Novartis, and research grants from AbbVie and Novartis. AMV received personal fees for advisory board and/or lectures from Novartis, AbbVie, AOP Pharmaceuticals, BMS and Incyte. All other authors have no conflict to report.

\section{ADDITIONAL INFORMATION}

Supplementary information The online version contains supplementary materia available at https://doi.org/10.1038/s41408-021-00581-6.

Correspondence and requests for materials should be addressed to Alessandro M. Vannucchi.

Reprints and permission information is available at http://www.nature.com/ reprints

Publisher's note Springer Nature remains neutral with regard to jurisdictional claims in published maps and institutional affiliations.

(i) Open Access This article is licensed under a Creative Commons Attribution 4.0 International License, which permits use, sharing, adaptation, distribution and reproduction in any medium or format, as long as you give appropriate credit to the original author(s) and the source, provide a link to the Creative Commons license, and indicate if changes were made. The images or other third party material in this article are included in the article's Creative Commons license, unless indicated otherwise in a credit line to the material. If material is not included in the article's Creative Commons license and your intended use is not permitted by statutory regulation or exceeds the permitted use, you will need to obtain permission directly from the copyright holder. To view a copy of this license, visit http://creativecommons. org/licenses/by/4.0/.

(c) The Author(s) 2021 